\title{
HARDY-POINCARÉ, RELLICH AND UNCERTAINTY PRINCIPLE INEQUALITIES ON RIEMANNIAN MANIFOLDS
}

\author{
ISMAIL KOMBE AND MURAD ÖZAYDIN
}

\begin{abstract}
We continue our previous study of improved Hardy, Rellich and uncertainty principle inequalities on a Riemannian manifold $M$, started in our earlier paper from 2009. In the present paper we prove new weighted Hardy-Poincaré, Rellich type inequalities as well as an improved version of our uncertainty principle inequalities on a Riemannian manifold $M$. In particular, we obtain sharp constants for these inequalities on the hyperbolic space $\mathbb{H}^{n}$.
\end{abstract}

\section{INTRODUCTION}

The classical Hardy, Rellich and Heisenberg-Pauli-Weyl (uncertainty principle) inequalities play important roles in many questions from spectral theory, harmonic analysis, partial differential equations, and geometry as well as quantum mechanics. In order to motivate our work, we present these three classical (sharp) inequalities on the Euclidean space $\mathbb{R}^{n}$. The Hardy inequality states that for $n \geq 3$

$$
\int_{\mathbb{R}^{n}}|\nabla \phi(x)|^{2} d x \geq\left(\frac{n-2}{2}\right)^{2} \int_{\mathbb{R}^{n}} \frac{|\phi(x)|^{2}}{|x|^{2}} d x,
$$

where $\phi \in C_{0}^{\infty}\left(\mathbb{R}^{n}\right)$. Here the constant $\left(\frac{n-2}{2}\right)^{2}$ is sharp, in the sense that

$$
\left(\frac{n-2}{2}\right)^{2}=\inf _{0 \neq \phi \in C_{0}^{\infty}\left(\mathbb{R}^{n}\right)} \frac{\int_{\mathbb{R}^{n}}|\nabla \phi(x)|^{2} d x}{\int_{\mathbb{R}^{n}} \frac{|\phi(x)|^{2}}{|x|^{2}} d x} .
$$

Another inequality involving second-order derivatives is the Rellich inequality [20]:

$$
\int_{\mathbb{R}^{n}}|\Delta \phi(x)|^{2} d x \geq \frac{n^{2}(n-4)^{2}}{16} \int_{\mathbb{R}^{n}} \frac{|\phi(x)|^{2}}{|x|^{4}} d x,
$$

where $\phi \in C_{0}^{\infty}\left(\mathbb{R}^{n}\right), n \geq 5$ and the constant $\frac{n^{2}(n-4)^{2}}{16}$ is again sharp. (There are also versions for lower dimensions under additional hypotheses.)

The classical Heisenberg-Pauli-Weyl inequality, a precise mathematical formulation of the uncertainty principle of quantum mechanics, states:

$$
\left(\int_{\mathbb{R}^{n}}|x|^{2}|f(x)|^{2} d x\right)\left(\int_{\mathbb{R}^{n}}|\nabla f(x)|^{2} d x\right) \geq \frac{n^{2}}{4}\left(\int_{\mathbb{R}^{n}}|f(x)|^{2} d x\right)^{2}
$$

Received by the editors May 6, 2011.

2010 Mathematics Subject Classification. Primary 26D10; Secondary 53C21.

Key words and phrases. Hardy-Poincaré inequality, Rellich inequality, uncertainty principle inequality. 
for all $f \in L^{2}\left(\mathbb{R}^{n}\right)$. Here the constant $\frac{n^{2}}{4}$ is sharp and also it is well known that equality is attained in (1.3) if and only if $f$ is Gaussian (i.e. $f(x)=A e^{-\alpha|x|^{2}}$ for some $A \in \mathbb{R}, \alpha>0)$.

These inequalities have been extensively studied in the Euclidean setting and now the literature on this topic is quite vast and rich, encompassing many generalizations and refinements, e.g. [2, [10, 6], [1], 3], [12, [8, [13] and the references therein. Many new developments are still forthcoming. For instance, Tertikas and Zographopoulos [22] give a sharp Rellich-type inequality and its improved versions which involves both first- and second-order derivatives:

$$
\int_{\mathbb{R}^{n}}|\Delta \phi(x)|^{2} d x \geq \frac{n^{2}}{4} \int_{\mathbb{R}^{n}} \frac{|\nabla \phi(x)|^{2}}{|x|^{2}} d x
$$

where $\phi \in C_{0}^{\infty}\left(\mathbb{R}^{n}\right), n \geq 5$ and the constant $\frac{n^{2}}{4}$ is sharp.

On the other hand the Euclidean results mentioned above continue to be a source of inspiration for the problem of finding analogous inequalities in the setting of Riemannian manifolds. There has been a continuously growing literature in this direction, e.g. [7, 9], 14, [18, 44, 23], 17], 19], and the references therein. For instance, in an interesting paper, Carron [7] obtained the following weighted $L^{2}$-Hardy inequality on a complete noncompact Riemannian manifold $M$ :

$$
\int_{M} \rho^{\alpha}|\nabla \phi|^{2} d V \geq\left(\frac{C+\alpha-1}{2}\right)^{2} \int_{M} \rho^{\alpha} \frac{\phi^{2}}{\rho^{2}} d V
$$

where $\phi \in C_{c}^{\infty}\left(M-\rho^{-1}\{0\}\right), \alpha \in \mathbb{R}, C>1, C+\alpha-1>0$ and the weight function $\rho$ satisfies $|\nabla \rho|=1$ and $\Delta \rho \geq \frac{C}{\rho}$ in the sense of distribution. For complete noncompact Riemannian manifolds, under the same geometric assumptions on the weight function $\rho$, we obtained in [17] an $L^{p}$ version of (1.5) (where $1<p<\infty$ and $C+1+\alpha-p>0)$

$$
\int_{M} \rho^{\alpha}|\nabla \phi|^{p} d V \geq\left(\frac{C+1+\alpha-p}{p}\right)^{p} \int_{M} \rho^{\alpha} \frac{|\phi|^{p}}{\rho^{p}} d V,
$$

as well as a Rellich-type inequality (where $\alpha<2, C+\alpha-3>0$ )

$$
\int_{M} \rho^{\alpha}|\Delta \phi|^{2} d V \geq \frac{(C+\alpha-3)^{2}(C-\alpha+1)^{2}}{16} \int_{M} \rho^{\alpha} \frac{\phi^{2}}{\rho^{4}} d V,
$$

where $\Delta$ is the Laplace-Beltrami operator on $M$.

We also found an $L^{p}$ Heisenberg-Pauli-Weyl uncertainty principle type inequality (for a complete noncompact Riemannian manifold) and an $L^{2}$ version with a (nonnegative) remainder term. In the specific case when the manifold $M$ is the hyperbolic space $\mathbb{H}^{n}$, we obtained sharp constants for the Hardy and Rellich-type inequalities, and explicit (not sharp) constants for the Heisenberg-Pauli-Weyl uncertainty inequalities.

In the present paper we continue our investigation of Hardy-Poincaré, Rellich and Heisenberg-Pauli-Weyl type inequalities. The plan of the paper is as follows. In Section 2 we first prove a new form of weighted Hardy-Poincaré type inequality and then we prove various improved versions of the weighted Hardy inequality (1.5) (in the sense that nonnegative terms are added on the right-hand side of (1.5)). We note that these improved inequalities are the main tool in proving improved Rellich type inequalities. In Section 3 we first prove a weighted analogue of (1.4) and then obtain improved versions. Section 4 is devoted to the study of 
Heisenberg-Pauli-Weyl (uncertainty principle) type inequalities where we obtain better constants than those of [17] and prove a sharp analogue of the classical uncertainty principle inequality (1.3) on the hyperbolic space $\mathbb{H}^{n}$. In each section we first prove inequalities in the context of a general complete Riemannian manifold. Then, turning our attention to the hyperbolic space $\mathbb{H}^{n}$, we consider specific weight functions and obtain inequalities with explicit and usually sharp constants.

\section{Weighted Hardy-Poincaré type inequalities}

Throughout this paper, $M$ denotes a complete noncompact Riemannian manifold endowed with a metric $g$. We denote by $d V, \nabla$, and $\Delta$ respectively the Riemannian volume element, the Riemannian gradient and the Laplace-Beltrami operator on $M$.

We begin this section by proving a new form of the Hardy-Poincaré type inequality for a complete noncompact Riemannian manifold $M$ with a weight function $\rho$ modelled on the distance from a point. (In this context the hypotheses $|\nabla \rho|=1$ and $\Delta \rho \geq \frac{C}{\rho}$ seem to be geometrically quite natural.) One advantage of this set-up is that it implies and thus provides another (shorter) proof of (1.6) above (Theorem 2.1 in [17]) as explained in the Remark below.

Theorem 2.1. Let $M$ be a complete noncompact Riemannian manifold of dimension $n>1$. Let $\rho$ be a nonnegative function on $M$ such that $|\nabla \rho|=1$ and $\Delta \rho \geq \frac{C}{\rho}$ in the sense of distribution where $C>0$. Then the following inequality holds:

$$
\int_{M} \rho^{\alpha+p}|\nabla \rho \cdot \nabla \phi|^{p} d V \geq\left(\frac{C+\alpha+1}{p}\right)^{p} \int_{M} \rho^{\alpha}|\phi|^{p} d V
$$

for all compactly supported smooth functions $\phi \in C_{0}^{\infty}\left(M \backslash \rho^{-1}\{0\}\right), 1<p<\infty$, and $C+\alpha>-1$.

Proof. It follows from the above hypothesis that

$$
\operatorname{div}(\rho \nabla \rho) \geq C+1 \text {. }
$$

Multiplying both sides of (2.2) by $\rho^{\alpha}|\phi|^{p}$ and integrating over $M$ yields

$$
(C+1) \int_{M} \rho^{\alpha}|\phi|^{p} d V \leq \int_{M} \operatorname{div}(\rho \nabla \rho) \rho^{\alpha}|\phi|^{p} d V .
$$

As an immediate consequence of the divergence theorem we have

$$
(C+\alpha+1) \int_{M} \rho^{\alpha}|\phi|^{p} d V \leq-p \int_{M}|\phi|^{p-2} \phi \rho^{\alpha+1} \nabla \rho \cdot \nabla \phi d V .
$$

An application of Hölder's and Young's inequalities yields

$$
\begin{aligned}
(C+ & \alpha+1) \int_{M} \rho^{\alpha}|\phi|^{p} d V \\
& \leq p\left(\int_{M} \rho^{\alpha}|\phi|^{p} d V\right)^{(p-1) / p}\left(\int_{M} \rho^{\alpha+p}|\nabla \rho \cdot \nabla \phi|^{p} d V\right)^{1 / p} \\
& \leq(p-1) \epsilon^{-p /(p-1)} \int_{M} \rho^{\alpha}|\phi|^{p} d V+\epsilon^{p} \int_{M} \rho^{\alpha+p}|\nabla \rho \cdot \nabla \phi|^{p} d V
\end{aligned}
$$

for any $\epsilon>0$. Therefore

$$
\int_{M} \rho^{\alpha+p}|\nabla \rho \cdot \nabla \phi|^{p} d V \geq \epsilon^{-p}\left(C+\alpha+1-(p-1) \epsilon^{-p /(p-1)}\right) \int_{M} \rho^{\alpha}|\phi|^{p} d V
$$


Note that the function $\epsilon \longrightarrow \epsilon^{-p}\left(C+\alpha+1-(p-1) \epsilon^{-p /(p-1)}\right)$ attains the maximum for $\epsilon^{p /(p-1)}=\frac{p}{C+\alpha+1}$, and this maximum is equal to $\left(\frac{C+\alpha+1}{p}\right)^{p}$. Now we obtain the desired inequality,

$$
\int_{M} \rho^{\alpha+p}|\nabla \rho \cdot \nabla \phi|^{p} d V \geq\left(\frac{C+\alpha+1}{p}\right)^{p} \int_{M} \rho^{\alpha}|\phi|^{p} d V
$$

Remark. Applying the Cauchy-Schwarz inequality to $|\nabla \rho \cdot \nabla \phi|$, replacing $\alpha$ with $\alpha-p$ and using $|\nabla \rho|=1$ yields the weighted $L^{p}$-Hardy inequality (1.6).

We will give a sharp version of Theorem 2.1 in the hyperbolic space $\mathbb{H}^{n}$. Recall that the hyperbolic space $\mathbb{H}^{n}(n \geq 2)$ is a complete simply connected Riemannian manifold having constant sectional curvature equal to -1 . There are several models for $\mathbb{H}^{n}$ and we will use the Poincaré ball model $\mathbb{B}^{n}$ in this paper.

The Poincaré ball model for the hyperbolic space is

$$
\mathbb{B}^{n}=\left\{x=\left(x_{1}, \ldots, x_{n}\right) \in \mathbb{R}^{n}|| x \mid<1\right\}
$$

endowed with the Riemannian metric $d s=\lambda(x)|d x|$, where $\lambda(x)=\frac{2}{1-|x|^{2}}$. Hence $\left\{\lambda d x_{i}\right\}_{i=1}^{n}$ give an orthonormal basis of the tangent space at $x=\left(x_{1}, \ldots, x_{n}\right)$ in $\mathbb{B}^{n}$. The corresponding dual basis is $\left\{\frac{1}{\lambda} \frac{\partial}{\partial x_{i}}\right\}_{i=1}^{n}$, thus the hyperbolic gradient and the Laplace-Beltrami operator are

$$
\begin{gathered}
\nabla_{\mathbb{H}^{n}} u=\frac{\nabla u}{\lambda}, \\
\Delta_{\mathbb{H}^{n}} u=\lambda^{-n} \operatorname{div}\left(\lambda^{n-2} \nabla u\right),
\end{gathered}
$$

where $\nabla$ and div denote the Euclidean gradient and divergence in $\mathbb{R}^{n}$, respectively.

The hyperbolic distance $d_{\mathbb{H}^{n}}(x, y)$ between $x, y \in \mathbb{B}^{n}$ in the Poincaré ball model is given by the formula

$$
d_{\mathbb{H}^{n}}(x, y)=\operatorname{Arccosh}\left(1+\frac{2|x-y|^{2}}{\left(1-|x|^{2}\right)\left(1-|y|^{2}\right)}\right) .
$$

From this we immediately obtain for $x \in \mathbb{B}^{n}$,

$$
d:=d_{\mathbb{H}^{n}}(0, x)=2 \operatorname{Arctanh}|x|=\log \left(\frac{1+|x|}{1-|x|}\right),
$$

which is the distance from $x \in \mathbb{B}^{n}$ to the origin. Moreover, the geodesic lines passing through the origin are the diameters of $\mathbb{B}^{n}$ along with open arcs of circles in $\mathbb{B}^{n}$ perpendicular to the boundary at $\infty, \partial \mathbb{B}^{n}=\mathbb{S}^{n-1}=\left\{x \in \mathbb{R}^{n}:|x|=1\right\}$.

The hyperbolic volume element is given by

$$
d V=\lambda^{n}(x) d x=\left(\frac{2}{1-r^{2}}\right)^{n} r^{n-1} d r d \sigma
$$

where $d x$ denotes the Lebesgue measure in $\mathbb{B}^{n}$ and $d \sigma$ is the normalized surface measure on $\mathbb{S}^{n-1}$. by

A hyperbolic ball in $\mathbb{B}^{n}$ with center 0 and hyperbolic radius $R \in(0, \infty)$ is defined

$$
B_{R}(0)=\left\{x \in \mathbb{B}^{n} \mid d_{\mathbb{H}^{n}}(0, x)<R\right\} ;
$$

we note that $B_{R}(0)$ is also a Euclidean ball with center 0 and radius $S=\tanh \frac{R}{2} \in$ $(0,1)$. 
Note that we have the following two relations for the distance function $d=$ $\log \left(\frac{1+|x|}{1-|x|}\right)$ :

$$
\begin{aligned}
\left|\nabla_{\mathbb{H}^{n}} d\right| & =1, \\
\Delta_{\mathbb{H}^{n}} d & \geq \frac{n-1}{d}, \quad x \neq 0 .
\end{aligned}
$$

We are now ready to give a sharp version of Theorem 2.1 in the hyperbolic space $\mathbb{H}^{n}$. Here $\rho$ is chosen to be the distance function from the origin in the Poincaré ball model for the hyperbolic space $\mathbb{H}^{n}$.

Theorem 2.2. Let $\phi \in C_{0}^{\infty}\left(\mathbb{H}^{n}\right), d=\log \left(\frac{1+|x|}{1-|x|}\right), n \geq 2,1<p<\infty$ and $\alpha>-n$. Then we have

$$
\int_{\mathbb{H}^{n}} d^{\alpha+p}\left|\nabla_{\mathbb{H}^{n}} d \cdot \nabla_{\mathbb{H}^{n}} \phi\right|^{p} d V \geq\left(\frac{n+\alpha}{p}\right)^{p} \int_{\mathbb{H}^{n}} d^{\alpha}|\phi|^{p} d V
$$

where the constant $\left(\frac{n+\alpha}{p}\right)^{p}$ is sharp.

Proof. The inequality follows from Theorem 2.1. We show that $\left(\frac{n+\alpha}{p}\right)^{p}$ is the best constant in (2.4):

$$
C_{H}:=\inf _{0 \neq \phi \in C_{0}^{\infty}\left(\mathbb{H}^{n}\right)} \frac{\int_{\mathbb{H}^{n}} d^{\alpha+p}\left|\nabla_{\mathbb{H}^{n}} d \cdot \nabla_{\mathbb{H}^{n}} \phi\right|^{p} d V}{\int_{\mathbb{H}^{n}} d^{\alpha}|\phi|^{p} d V}=\left(\frac{n+\alpha}{p}\right)^{p} .
$$

It is clear that

$$
\left(\frac{n+\alpha}{p}\right)^{p} \leq \frac{\int_{\mathbb{H}^{n}} d^{\alpha+p}\left|\nabla_{\mathbb{H}^{n}} d \cdot \nabla_{\mathbb{H}^{n}} \phi\right|^{p} d V}{\int_{\mathbb{H}^{n}} d^{\alpha}|\phi|^{p} d V}
$$

holds for all $\phi \in C_{0}^{\infty}\left(\mathbb{H}^{n}\right)$. If we pass to the inf in (2.5) we get that $\left(\frac{n+\alpha}{p}\right)^{p} \leq C_{H}$. We only need to show that $C_{H} \leq\left(\frac{n+\alpha}{p}\right)^{p}$ and for this we use the family of radial functions

$$
\phi_{\epsilon}(d)= \begin{cases}d^{\frac{n+\alpha}{p}+\epsilon} & \text { if } \quad d \in[0,1], \\ d^{-\left(\frac{n+\alpha}{p}+\epsilon\right)} & \text { if } \quad d>1,\end{cases}
$$

where $\epsilon>0$. Notice that $\phi_{\epsilon}(d)$ can be approximated by smooth functions with compact support in $\mathbb{H}^{n}$.

A direct computation shows that

$$
d^{\alpha+p}\left|\nabla_{\mathbb{H}^{n}} d \cdot \nabla_{\mathbb{H}^{n}} \phi_{\epsilon}\right|^{p}= \begin{cases}\left(\frac{n+\alpha}{p}+\epsilon\right)^{p} d^{n+2 \alpha+p \epsilon} & \text { if } d \in[0,1], \\ \left(\frac{n+\alpha}{p}+\epsilon\right)^{p} d^{-n-p \epsilon} & \text { if } d>1 .\end{cases}
$$

Let us denote by $\mathbb{B}_{1}=\left\{x \in \mathbb{H}^{n}: d \leq 1\right\}$ the unit ball with respect to the distance $d$. Hence

$$
\int_{\mathbb{H}^{n}} d^{\alpha}\left|\phi_{\epsilon}\right|^{p} d V=\int_{\mathbb{B}_{1}} d^{n+2 \alpha+p \epsilon} d V+\int_{\mathbb{H}^{n} \backslash \mathbb{B}_{1}} d^{-n-p \epsilon} d V
$$

and then we have

$$
\begin{aligned}
\left(\frac{n+\alpha}{p}+\epsilon\right)^{p} \int_{\mathbb{H}^{n}} d^{\alpha}\left|\phi_{\epsilon}\right|^{p} d V & =\left(\frac{n+\alpha}{p}+\epsilon\right)^{p}\left[\int_{\mathbb{B}_{1}} d^{n+2 \alpha+p \epsilon} d V+\int_{\mathbb{H}^{n} \backslash \mathbb{B}_{1}} d^{-n-p \epsilon} d V\right] \\
& =\int_{\mathbb{H}^{n}} d^{\alpha+p}\left|\nabla_{\mathbb{H}^{n}} d \cdot \nabla_{\mathbb{H}^{n}} \phi_{\epsilon}\right|^{p} d V .
\end{aligned}
$$


On the other hand,

$$
\begin{aligned}
\frac{\left(\frac{n+\alpha}{p}+\epsilon\right)^{p}}{C_{H}} \int_{\mathbb{H}^{n}} d^{\alpha+p}\left|\nabla_{\mathbb{H}^{n}} d \cdot \nabla_{\mathbb{H}^{n}} \phi_{\epsilon}\right|^{p} d V & \geq\left(\frac{n+\alpha}{p}+\epsilon\right)^{p} \int_{\mathbb{H}^{n}} d^{\alpha}\left|\phi_{\epsilon}\right|^{p} d V \\
& =\int_{\mathbb{H}^{n}} d^{\alpha+p}\left|\nabla_{\mathbb{H}^{n}} d \cdot \nabla_{\mathbb{H}^{n}} \phi_{\epsilon}\right|^{p} d V .
\end{aligned}
$$

It is clear that $\left(\frac{n+\alpha}{p}+\epsilon\right)^{p} \geq C_{H}$, and letting $\epsilon \longrightarrow 0$, we obtain $\left(\frac{n+\alpha}{p}\right)^{p} \geq C_{H}$. Therefore $C_{H}=\left(\frac{n+\alpha}{p}\right)^{p}$.

We now prove an improved $L^{2}$ weighted Hardy inequality involving two weight functions $\rho$ and $\delta$ modeled on distance functions from a point and distance to the boundary of a domain $\Omega$ with smooth boundary.

Theorem 2.3. Let $M$ be a complete noncompact Riemannian manifold of dimension $n>1$. Let $\rho$ and $\delta$ be nonnegative functions on $M$ such that $|\nabla \rho|=1, \Delta \rho \geq \frac{C}{\rho}$ and $-\operatorname{div}\left(\rho^{1-C} \nabla \delta\right) \geq 0$ in the sense of distribution, where $C>1$. Then we have

$$
\int_{\Omega} \rho^{\alpha}|\nabla \phi|^{2} d V \geq\left(\frac{C+\alpha-1}{2}\right)^{2} \int_{\Omega} \rho^{\alpha} \frac{\phi^{2}}{\rho^{2}} d V+\frac{1}{4} \int_{\Omega} \rho^{\alpha} \frac{|\nabla \delta|^{2}}{\delta^{2}} \phi^{2} d V
$$

for all $\phi \in C_{0}^{\infty}\left(\Omega \backslash \rho^{-1}\{0\}\right), \alpha \in \mathbb{R}$, and $C+\alpha-1>0$.

Proof. Let $\phi \in C_{0}^{\infty}$ and define $\psi=\rho^{\beta} \phi$, where $\beta<0$. A direct calculation shows that

$$
|\nabla \phi|^{2}=\beta^{2} \rho^{2 \beta-2}|\nabla \rho|^{2} \psi^{2}+2 \beta \rho^{2 \beta-1} \psi \nabla \rho \cdot \nabla \psi+\rho^{2 \beta}|\nabla \psi|^{2} .
$$

Multiplying both sides of (2.8) by $\rho^{\alpha}$ and applying integration by parts over $M$ gives

$$
\begin{aligned}
\int_{M} \rho^{\alpha}|\nabla \phi|^{2} d V & =\beta^{2} \int_{M} \rho^{\alpha+2 \beta-2} \psi^{2} d V-\frac{\beta}{\alpha+2 \beta} \int_{M} \Delta\left(\rho^{\alpha+2 \beta}\right) \psi^{2} d V \\
& +\int_{M} \rho^{\alpha+2 \beta}|\nabla \psi|^{2} d V \\
& \geq-\beta^{2}-\beta(\alpha+C-1) \int_{M} \rho^{\alpha-2} \phi^{2} d V+\int_{M} \rho^{\alpha+2 \beta}|\nabla \psi|^{2} d V .
\end{aligned}
$$

Choosing

$$
\beta=\frac{1-\alpha-C}{2}
$$

gives

$$
\int_{M} \rho^{\alpha}|\nabla \phi|^{2} d V \geq \int_{M} \rho^{\alpha} \frac{\phi^{2}}{\rho^{2}} d V+\int_{M} \rho^{1-C}|\nabla \psi|^{2} d V .
$$

We now focus on the second term on the right-hand side of this inequality. Let us define a new variable $\varphi(x):=\delta(x)^{-1 / 2} \psi(x)$, where $\delta(x)$ is a nonnegative function and $\delta(x) \in C_{0}^{2}(M)$. It is clear that

$$
|\nabla \psi|^{2}=\frac{1}{4} \frac{\varphi^{2}}{\delta}|\nabla \delta|^{2}+\varphi \nabla \delta \cdot \nabla \varphi+\delta|\nabla \varphi|^{2} .
$$


Therefore

$$
\begin{aligned}
\int_{M} \rho^{1-C}|\nabla \psi|^{2} d V & \geq \frac{1}{4} \int_{M} \rho^{1-C} \frac{\varphi^{2}}{\delta}|\nabla \delta|^{2} d V+\int_{M} \rho^{1-C} \varphi \nabla \delta \cdot \nabla \varphi d V \\
& =\frac{1}{4} \int_{M} \rho^{1-C} \frac{|\nabla \delta|^{2}}{\delta^{2}} \psi^{2} d V-\frac{1}{2} \int_{M} \operatorname{div}\left(\rho^{1-C} \nabla \delta\right) \varphi^{2} d V
\end{aligned}
$$

Since $-\operatorname{div}\left(\rho^{1-C} \nabla \delta\right) \geq 0$ and $\psi=\rho^{\frac{C+\alpha-1}{2}} \phi$, then we get

$$
\int_{M} \rho^{1-C}|\nabla \psi|^{2} d V \geq \frac{1}{4} \int_{M} \rho^{\alpha} \frac{|\nabla \delta|^{2}}{\delta^{2}} \phi^{2} d V
$$

Substituting (2.11) into (2.10) gives the desired inequality:

$$
\int_{M} \rho^{\alpha}|\nabla \phi|^{2} d V \geq\left(\frac{C+\alpha-1}{2}\right)^{2} \int_{M} \rho^{\alpha} \frac{\phi^{2}}{\rho^{2}} d V+\frac{1}{4} \int_{M} \rho^{\alpha} \frac{|\nabla \delta|^{2}}{\delta^{2}} \phi^{2} d V .
$$

Our next goal is to find model functions which satisfy the assumption of the above theorem. A straightforward computation shows that $\delta=\log \left(\frac{R}{\rho}\right)$ satisfies the differential inequality $-\operatorname{div}\left(\rho^{1-C} \nabla \delta\right) \geq 0$. As a consequence of Theorem 2.3 we have the following weighted $L^{2}$-Hardy-type inequality on the hyperbolic space $\mathbb{H}^{n}$ which has a logarithmic remainder term. The sharpness of the constant $\left(\frac{n+\alpha-2}{2}\right)^{2}$ follows as in [17, Theorem 3.1].

Corollary 2.1. Let $\Omega$ be a bounded domain with smooth boundary $\partial \Omega$ in $\mathbb{H}^{n}$. Let $\rho=d=\log \left(\frac{1+|x|}{1-|x|}\right)$ and $\delta:=\log \left(\frac{R}{d}\right), R>\sup _{\Omega}(d), \alpha \in \mathbb{R}, n+\alpha-2>0$. Then we have

$$
\int_{\Omega} d^{\alpha}\left|\nabla_{\mathbb{H}^{n}} \phi\right|^{2} d V \geq\left(\frac{n+\alpha-2}{2}\right)^{2} \int_{\Omega} d^{\alpha} \frac{\phi^{2}}{d^{2}} d V+\frac{1}{4} \int_{\Omega} d^{\alpha} \frac{\phi^{2}}{d^{2}\left(\log \frac{R}{d}\right)^{2}} d V
$$

for all $\phi \in C_{0}^{\infty}(\Omega)$ and the constant $\left(\frac{n+\alpha-2}{2}\right)^{2}$ is sharp.

Let $B_{R}=\left\{x \in \mathbb{B}^{n} \mid d<R\right\}$ be a hyperbolic ball with center 0 and hyperbolic radius $R$. It is clear that $\delta:=R-d$ is the distance function of the point $x \in B_{R}$ to the boundary of $B_{R}$ and satisfies the differential inequality in Theorem 2.4. Therefore we have

Corollary 2.2. Let $B_{R}$ be a hyperbolic ball with center 0 and hyperbolic radius $R$. Let $d=\log \left(\frac{1+|x|}{1-|x|}\right)$ and $\delta:=R-d, \alpha \in \mathbb{R}, n+\alpha-2>0$. Then we have

$$
\int_{B_{R}} d^{\alpha}\left|\nabla_{\mathbb{H}^{n}} \phi\right|^{2} d V \geq\left(\frac{n+\alpha-2}{2}\right)^{2} \int_{B_{R}} d^{\alpha} \frac{\phi^{2}}{d^{2}} d V+\frac{1}{4} \int_{B_{R}} d^{\alpha} \frac{\phi^{2}}{(R-d)^{2}} d V
$$

for all $\phi \in C_{0}^{\infty}\left(B_{R}\right)$ and the constant $\left(\frac{n+\alpha-2}{2}\right)^{2}$ is sharp.

Hardy-Sobolev-Poincaré inequalities. The following sharp form of the Sobolev inequality on the hyperbolic space $\mathbb{H}^{n}$ is due to [16. It states that for all $\phi \in$ $C_{0}^{\infty}\left(\mathbb{H}^{n}\right)$ :

$$
\int_{\mathbb{H}^{n}}\left|\nabla_{\mathbb{H}^{n}} \phi\right|^{2} d V \geq \frac{n(n-2)}{4}\left|\mathbb{S}^{n}\right|^{\frac{2}{n}}\left(\int_{\mathbb{H}^{n}}|\phi|^{\frac{2 n}{n-2}} d V\right)^{\frac{n-2}{n}}+\frac{n(n-2)}{4} \int_{\mathbb{H}^{n}} \phi^{2} d V
$$

where $\phi \in C_{0}^{\infty}\left(\mathbb{H}^{n}\right)$. Here $A_{n}=\frac{n(n-2)}{4}|\mathbb{S}|^{\frac{2}{n}}$ is the sharp constant for the Sobolev inequality on $\mathbb{R}^{n},\left|\mathbb{S}^{n}\right|$ is the volume of the $n$-dimensional unit sphere in $\mathbb{R}^{n+1}$ and 
the constant $B_{n}=\frac{n(n-2)}{4}$ is sharp for $n \geq 4$. Recently, sharp form of the inequality (2.14) in three-dimensional hyperbolic space $\mathbb{H}^{n}$ has been proved by Benguria, Frank and Loss in [5].

The Sobolev inequality (2.14) and the Hardy inequality in [17] yield the following Hardy-Sobolev inequality on the hyperbolic space $\mathbb{H}^{n}$.

Corollary 2.3. Let $\phi \in C_{0}^{\infty}\left(\mathbb{H}^{n}\right), d=\log \left(\frac{1+|x|}{1-|x|}\right)$ and $n \geq 3$. Then we have

$$
\int_{\mathbb{H}^{n}}\left|\nabla_{\mathbb{H}^{n}} \phi\right|^{2} d V \geq\left(\frac{n-2}{2}\right)^{\frac{2 s}{p^{*}(s)}}\left(\frac{n(n-2)}{4}\left|\mathbb{S}^{n}\right|^{\frac{2}{n}}\right)^{\frac{n(2-s)}{2(n-s)}}\left(\int_{\mathbb{H}^{n}} \frac{|\phi|^{p^{*}(s)}}{d^{s}} d V\right)^{\frac{2}{p^{*}(s)}},
$$

where $0 \leq s \leq 2$ and $p^{*}(s)=2\left(\frac{n-s}{n-2}\right)$.

Before we state and prove our next theorem, we first recall the (Euclidean) weighted Sobolev inequality of Fabes, Kenig and Serapino [11, which plays an important role in our proof. They proved the following inequality:

$$
\left(\frac{1}{w\left(B_{r}\right)} \int_{B_{r}}|\nabla \phi|^{p} w(x) d x\right)^{1 / p} \geq \frac{1}{c\left(\operatorname{diam} B_{r}\right)}\left(\frac{1}{w\left(B_{r}\right)} \int_{B_{r}}|\phi|^{k p} w(x) d x\right)^{1 / k p},
$$

where $B_{r}$ is a ball in $\mathbb{R}^{n}, \phi \in C_{0}^{\infty}\left(B_{r}\right), w\left(B_{r}\right)=\int_{B_{r}} w(x) d x, 1<p<\infty, 1 \leq$ $k \leq \frac{n}{n-1}+\epsilon, \epsilon>0$ and the weight function $w$ belongs to Muckenhoupt's class $A_{p}$. In particular, if the weight function $w$ belongs to Muckenhoupt's class $A_{2}$, then $k$ can be taken equal to $\frac{n}{n-1}+\epsilon$ and this is sharp. Recall that a weight function $w$ belongs to Muckenhoupt's class $A_{p}(1<p<\infty)$ if

$$
\sup \left(\frac{1}{|B|} \int_{B} w(x) d x\right)\left(\frac{1}{|B|} \int_{B} w(x)^{\frac{1}{1-p}} d x\right)^{p-1}=C_{p, w}<\infty,
$$

where the supremum is taken over all balls $B$ in $\mathbb{R}^{n}$ (see [21]).

Motivated by the classical work of Brezis and Vázquez [6], our next theorem shows that a sharp weighted Hardy inequality on the hyperbolic space $\mathbb{H}^{n}$ can be improved by a weighted Sobolev term.

Theorem 2.4. Let $\phi \in C_{0}^{\infty}\left(\mathbb{H}^{n}\right), d=\log \left(\frac{1+|x|}{1-|x|}\right), \alpha \in \mathbb{R}, n>2$ and $n+\alpha-2>0$. Then we have

$$
\int_{\mathbb{H}^{n}} d^{\alpha}\left|\nabla_{\mathbb{H}^{n}} \phi\right|^{2} d V \geq\left(\frac{n+\alpha-2}{2}\right)^{2} \int_{\mathbb{H}^{n}} d^{\alpha} \frac{\phi^{2}}{d^{2}} d V+\tilde{c}\left(\int_{\mathbb{H}^{n}} d^{\frac{(2-n)(2-q)+\alpha q}{2}} \phi^{q} d x\right)^{2 / q},
$$

where $2 \leq q \leq \frac{2 n}{n-1}+2 \epsilon, \epsilon>0, \tilde{c}=\frac{2^{n-2}}{c^{2}}\left(\frac{\left|\mathbb{S}^{n}\right|}{2}\right)^{\frac{q-2}{q}}, c>0$ and the constant $\left(\frac{n+\alpha-2}{2}\right)^{2}$ is sharp.

Proof. Let $\phi \in C_{0}^{\infty}$ and define $\psi=d^{-\beta} \phi$, where $\beta<0$. A direct calculation shows that

$$
\begin{aligned}
d^{\alpha}|\nabla \phi|^{2} \lambda^{n-2}= & \beta^{2} d^{\alpha+2 \beta-2}|\nabla d|^{2} \psi^{2} \lambda^{n-2} \\
& +2 \beta d^{\alpha+2 \beta-1} \psi \lambda^{n-2} \nabla d \cdot \nabla \psi+d^{\alpha+2 \beta}|\nabla \psi|^{2} \lambda^{n-2} .
\end{aligned}
$$

It is easy to see that

$$
|\nabla d|^{2}=\lambda^{2}
$$


and integrating (2.17) over $\mathbb{B}^{n}$, we get

(2.18)

$$
\begin{aligned}
\int_{\mathbb{B}^{n}} d^{\alpha}|\nabla \phi|^{2} \lambda^{n-2} d x & =\int_{\mathbb{B}^{n}} \beta^{2} d^{\alpha+2 \beta-2} \psi^{2} \lambda^{n} d x+\int_{\mathbb{B}^{n}} 2 \beta d^{\alpha+2 \beta-1} \psi \lambda^{n-2} \nabla d \cdot \nabla \psi d x \\
& +\int_{\mathbb{B}^{n}} d^{\alpha+2 \beta}|\nabla \psi|^{2} \lambda^{n-2} d x .
\end{aligned}
$$

Applying integration by parts to the middle integral on the right-hand side of (2.18), we obtain

$$
\begin{aligned}
\int_{\mathbb{B}^{n}} d^{\alpha}|\nabla \phi|^{2} \lambda^{n-2} d x & =\int_{\mathbb{B}^{n}} \beta^{2} d^{\alpha+2 \beta-2} \psi^{2} \lambda^{n} d x-\frac{\beta}{\alpha+2 \beta} \int_{\mathbb{B}^{n}} \operatorname{div}\left(\lambda^{n-2} \nabla\left(d^{2 \beta+\alpha}\right)\right) d x \\
& +\int_{\mathbb{B}^{n}} d^{\alpha+2 \beta}|\nabla \psi|^{2} \lambda^{n-2} d x
\end{aligned}
$$

One can show that

$$
\begin{aligned}
& -\frac{\beta}{\alpha+2 \beta} \int_{\mathbb{B}^{n}} \operatorname{div}\left(\lambda^{n-2} \nabla\left(d^{2 \beta+\alpha}\right)\right) d x \\
= & -\beta(2 \beta+\alpha-1) \int_{\mathbb{B}^{n}} d^{2 \beta+\alpha-2} \lambda^{n} \psi^{2} d x-\beta \int_{\mathbb{B}^{n}} d^{2 \beta+\alpha-1} \lambda^{n-2} \psi^{2}(\Delta d) d x \\
& -\beta(n-2) \int_{\mathbb{B}^{n}} d^{2 \beta+\alpha-1} \lambda^{n-3}(\nabla d \cdot \nabla \lambda) d x .
\end{aligned}
$$

A direct computation shows that

and

$$
\Delta d=\lambda^{2} r+\frac{n-1}{r} \lambda
$$

$$
\nabla d \cdot \nabla \lambda=\lambda^{3} r
$$

Substituting these above

$$
\begin{aligned}
& -\frac{\beta}{\alpha+2 \beta} \int_{\mathbb{B}^{n}} \operatorname{div}\left(\lambda^{n-2} \nabla\left(d^{2 \beta+\alpha}\right)\right) d x \\
& =-\beta(2 \beta+\alpha-1) \int_{\mathbb{B}^{n}} d^{2 \beta+\alpha-2} \lambda^{n} \psi^{2} d x \\
& \quad-(2 \beta+\alpha) \int_{\mathbb{B}^{n}} d^{2 \beta+\alpha-1} \lambda^{n}\left(\frac{(n-1)\left(\lambda r^{2}+1\right)}{\lambda r}\right) \psi^{2} d x .
\end{aligned}
$$

We can easily show that

If $2 \beta+\alpha<0$, then we have

$$
\frac{\lambda r^{2}+1}{\lambda r} \geq \frac{1}{d}
$$

$$
-\frac{\beta}{\alpha+2 \beta} \int_{\mathbb{B}^{n}} \operatorname{div}\left(\lambda^{n-2} \nabla\left(d^{2 \beta+\alpha}\right)\right) d x \geq-\beta(2 \beta+\alpha+n-2) \int_{\mathbb{B}^{n}} d^{2 \beta+\alpha-2} \lambda^{n} \psi^{2} d x .
$$

Now we substitute (2.22) into (2.19) and we get

$$
\begin{aligned}
\int_{\mathbb{B}^{n}} d^{\alpha}|\nabla \phi|^{2} \lambda^{n-2} \geq & \left(-\beta^{2}-\beta(\alpha+n-2)\right) \int_{\mathbb{B}^{n}} d^{2 \beta+\alpha-2} \psi^{2} \lambda^{n} d x \\
& +\int_{\mathbb{B}^{n}} d^{\alpha+2 \beta}|\nabla \psi|^{2} \lambda^{n-2} d x .
\end{aligned}
$$


Note that the function $\beta \longrightarrow-\beta^{2}-\beta(\alpha+n-2)$ attains the maximum for $\beta=\frac{2-\alpha-n}{2}$, and this maximum is equal to $\left(\frac{n+\alpha-2}{2}\right)^{2}$. Therefore we have the following inequality:

$$
\int_{\mathbb{B}^{n}} d^{\alpha}|\nabla \phi|^{2} \lambda^{n-2} d x \geq\left(\frac{n+\alpha-2}{2}\right)^{2} \int_{\mathbb{B}^{n}} d^{\alpha} \frac{\phi^{2}}{d^{2}} \lambda^{n} d x+\int_{\mathbb{B}^{n}} d^{2-n}|\nabla \psi|^{2} \lambda^{n-2} d x .
$$

Using the fact $d \leq \lambda r$, we get

$$
\int_{\mathbb{B}^{n}} d^{\alpha}|\nabla \phi|^{2} \lambda^{n-2} d x \geq\left(\frac{n+\alpha-2}{2}\right)^{2} \int_{\mathbb{B}^{n}} d^{\alpha} \frac{\phi^{2}}{d^{2}} \lambda^{n} d x+\int_{\mathbb{B}^{n}} r^{2-n}|\nabla \psi|^{2} d x .
$$

Notice that the weight function $r^{2-n}$ is in the Muckenhoupt $A_{2}$ class. We now apply weighted Sobolev inequality (2.15) to the second integral term on the right-hand side of (2.23) and obtain

$$
\begin{aligned}
\int_{\mathbb{B}^{n}} d^{\alpha}|\nabla \phi|^{2} \lambda^{n-2} d x \\
\quad \geq\left(\frac{n+\alpha-2}{2}\right)^{2} \int_{\mathbb{B}^{n}} d^{\alpha} \frac{\phi^{2}}{d^{2}} \lambda^{n} d x+c_{1}\left(\int_{\mathbb{B}^{n}} r^{2-n} \psi^{q} d x\right)^{2 / q} \\
\quad \geq\left(\frac{n+\alpha-2}{2}\right)^{2} \int_{\mathbb{B}^{n}} d^{\alpha} \frac{\phi^{2}}{d^{2}} \lambda^{n} d x+c_{1}\left(\int_{\mathbb{B}^{n}} r^{2-n} d^{\frac{(n+\alpha-2) q}{2}} \phi^{q} d x\right)^{2 / q},
\end{aligned}
$$

where $q>2$ and $c_{1}=\frac{1}{c^{2}}\left(\frac{\left|\mathbb{S}^{n}\right|}{2}\right)^{1-\frac{1}{k}}$. Furthermore, using the inequality $2 r \leq d \leq \lambda r$, we get

$\int_{\mathbb{B}^{n}} d^{\alpha}|\nabla \phi|^{2} \lambda^{n-2} d x \geq\left(\frac{n+\alpha-2}{2}\right)^{2} \int_{\mathbb{B}^{n}} d^{\alpha} \frac{\phi^{2}}{d^{2}} \lambda^{n} d x+\tilde{c}\left(\int_{\mathbb{B}^{n}} d^{\frac{(n-2)(q-2)+\alpha q}{2}} \phi^{q} d x\right)^{2 / q}$, where $q>2$ and $\tilde{c}=\frac{2^{n-2}}{c^{2}}\left(\frac{\left|\mathbb{S}^{n}\right|}{2}\right)^{\frac{q-2}{q}}$. This completes the proof.

\section{RELLICH-TYPE INEQUALITIES}

In this section we prove the weighted Rellich-type inequality and its improved versions, which connects first to the second-order derivatives. The following is the weighted analogue of (1.4) in the setting of Riemannian manifold $M$.

Theorem 3.1. Let $M$ be a complete Riemannian manifold of dimension $n>1$. Let $\rho$ be a nonnegative function on $M$ such that $|\nabla \rho|=1$ and $\Delta \rho \geq \frac{C}{\rho}$ in the sense of distribution where $C>1$. Then the following inequality is valid:

$$
\int_{M} \rho^{\alpha}|\Delta \phi|^{2} d V \geq \frac{(C+1-\alpha)^{2}}{4} \int_{M} \rho^{\alpha} \frac{|\nabla \phi|^{2}}{\rho^{2}} d V
$$

for all compactly supported smooth functions $\phi \in C_{0}^{\infty}\left(M \backslash \rho^{-1}\{0\}\right), \frac{7-C}{3}<\alpha<2$.

Proof. A straightforward computation shows that

$$
\Delta \rho^{\alpha-2} \leq(\alpha-2)(C+\alpha-3) \rho^{\alpha-4} .
$$

Multiplying both sides of (3.2) by $\phi^{2}$ and integrating over $M$, we obtain

$$
\begin{aligned}
(C+\alpha-3)(\alpha-2) \int_{M} \rho^{\alpha-4} \phi^{2} d V & \geq \int_{M} \rho^{\alpha-2} \Delta\left(\phi^{2}\right) d V \\
& =\int_{M} \rho^{\alpha-2}\left(2|\nabla \phi|^{2}+2 \phi \Delta \phi\right) d V .
\end{aligned}
$$


Therefore

$$
-\int_{M}(\phi \Delta \phi) \rho^{\alpha-2} \geq \int_{M} \rho^{\alpha-2}|\nabla \phi|^{2} d V-\frac{(C+\alpha-3)(\alpha-2)}{2} \int_{M} \rho^{\alpha-4} \phi^{2} d V .
$$

Let us apply Young's inequality to expression $-\int_{M} \rho^{\alpha-2} \phi \Delta \phi d x$,

$$
-\int_{M} \rho^{\alpha-2} \phi \Delta \phi d V \leq \epsilon \int_{M} \rho^{\alpha-4} \phi^{2} d V+\frac{1}{4 \epsilon} \int_{M} \rho^{\alpha}|\Delta \phi|^{2} d V,
$$

where $\epsilon>0$ and will be chosen later. Combining (3.5) and (3.4), we get

$$
\int_{M} \rho^{\alpha-2}|\nabla \phi|^{2} d V \leq\left(\epsilon+\frac{(C+\alpha-3)(\alpha-2)}{2}\right) \int_{M} \rho^{\alpha-4} \phi^{2} d V+\frac{1}{4 \epsilon} \int_{M} \rho^{\alpha}|\Delta \phi|^{2} d V .
$$

Notice that the case of $\epsilon+\frac{(C+\alpha-3)(\alpha-2)}{2}<0$ gives the Rellich inequality (1.6). Therefore we only need to consider the cases $\epsilon+\frac{(C+\alpha-3)(\alpha-2)}{2}=0$ and $\epsilon+$ $\frac{(C+\alpha-3)(\alpha-2)}{2}>0$, respectively. The first case gives the following inequality:

$$
\int_{M} \rho^{\alpha}|\Delta \phi|^{2} d V \geq 2(C+\alpha-3)(2-\alpha) \int_{M} \rho^{\alpha-2}|\nabla \phi|^{2} d V
$$

If $\epsilon+\frac{(C+\alpha-3)(\alpha-2)}{2}>0$, then we apply the Rellich inequality (1.6) to the first term on the right-hand side of (3.6) and get

$$
\int_{M} \rho^{\alpha-2}|\nabla \phi|^{2} d V \leq P_{C, \alpha}(\epsilon) \int_{M} \rho^{\alpha}|\Delta \phi|^{2} d V
$$

where

$$
P_{C, \alpha}(\epsilon)=\frac{16 \epsilon}{(C+\alpha-3)^{2}(C-\alpha+1)^{2}}+\frac{8(\alpha-2)}{(C+\alpha-3)(C-\alpha+1)^{2}}+\frac{1}{4 \epsilon} .
$$

Note that the function $P_{C, \alpha}(\epsilon)$ attains the minumum for $\epsilon=\frac{(C+\alpha-3)(C-\alpha+1)}{8}$, and this minimum is equal to $\frac{4}{(C-\alpha+1)^{2}}$. Therefore we have the following inequality:

$$
\int_{M} \rho^{\alpha}|\Delta \phi|^{2} d V \geq \frac{(C-\alpha+1)^{2}}{4} \int_{M} \rho^{\alpha-2}|\nabla \phi|^{2} d V
$$

We are now ready to give a sharp version of Theorem 3.1 in the hyperbolic space $\mathbb{H}^{n}$. Here $\rho$ is chosen to be the distance function from the origin in the Poincare ball model for the hyperbolic space.

Theorem 3.2. Let $\phi \in C_{0}^{\infty}\left(\mathbb{H}^{n}\right), d=\log \left(\frac{1+|x|}{1-|x|}\right), n>2, \frac{8-n}{3}<\alpha<2$. Then we have

$$
\int_{\mathbb{H}^{n}} d^{\alpha}\left|\Delta_{\mathbb{H}^{n}} \phi\right|^{2} d V \geq \frac{(n-\alpha)^{2}}{4} \int_{\mathbb{H}^{n}} d^{\alpha} \frac{\left|\nabla_{\mathbb{H}^{n}} \phi\right|^{2}}{d^{2}} d V .
$$

Proof. The inequality follows from Theorem 3.1. To show that the constant $\left(\frac{n-\alpha}{2}\right)^{2}$ is sharp, we use the following family of functions as in [17]:

$$
\phi_{\epsilon}(d)= \begin{cases}-\left(\frac{n+\alpha-4}{2}+\epsilon\right)(d-1)+1 & \text { if } d \in[0,1], \\ d^{-\left(\frac{n+\alpha-4}{2}+\epsilon\right)} & \text { if } \quad d>1 .\end{cases}
$$


Notice that $\phi_{\epsilon}(d)$ can be well approximated by smooth functions with compact support in $\mathbb{H}^{n}$, and direct computation shows that $\frac{(n-\alpha)^{2}}{4}$ is the best constant in $(3.9)$ :

$$
\frac{(n-\alpha)^{2}}{4}=\lim _{\epsilon \longrightarrow 0} \frac{\int_{\mathbb{H}^{n}} d^{\alpha}\left|\Delta_{\mathbb{H}^{n}} \phi_{\epsilon}\right|^{2} d V}{\int_{\mathbb{H}^{n}} d^{\alpha} \frac{\mid \nabla_{\left.\mathbb{H}^{n} \phi_{\epsilon}\right|^{2}}}{d^{2}} d V} .
$$

The following inequality is an improved version of the Rellich-type inequality (3.1) for bounded domains.

Theorem 3.3. Let $\Omega$ be a bounded domain with smooth boundary $\partial \Omega$ in a complete Riemannian manifold of dimension $n>1$. Let $\rho$ be a nonnegative function on $M$ such that $|\nabla \rho|=1, \Delta \rho \geq \frac{C}{\rho}$ and $-\operatorname{div}\left(\rho^{1-C} \nabla \delta\right) \geq 0$ in the sense of distribution, where $C>1$. Then the following inequality is valid:

$$
\int_{\Omega} \rho^{\alpha}|\Delta \phi|^{2} d V \geq \frac{(C+1-\alpha)^{2}}{4} \int_{\Omega} \rho^{\alpha} \frac{|\nabla \phi|^{2}}{\rho^{2}} d V+K(C, \alpha) \int_{\Omega} \rho^{\alpha-2} \frac{|\nabla \delta|^{2}}{\delta^{2}} \phi^{2} d V
$$

for all compactly supported smooth functions $\phi \in C_{0}^{\infty}\left(M \backslash \rho^{-1}\{0\}\right), \frac{7-C}{3}<\alpha<2$ and $K(C, \alpha)=\frac{(C+1-\alpha)(C+3 \alpha-7)}{16}$.

Proof. The proof is similar to the proof of Theorem 3.1. The only difference is that we apply the improved Hardy-type inequality (2.7) to the first term on the right-hand side of (3.6).

The following corollaries are the direct consequences of Theorem 3.3.

Corollary 3.1. Let $\Omega$ be a bounded domain with smooth boundary in $\mathbb{H}^{n}$. Let $d=\log \left(\frac{1+|x|}{1-|x|}\right)$ and $\delta:=\log \left(\frac{R}{d}\right)$ and $R>\sup _{\Omega}(d)$. Then the following inequality is valid:

$$
\int_{\Omega} d^{\alpha}\left|\Delta_{\mathbb{H}^{n}} \phi\right|^{2} d V \geq \frac{(n-\alpha)^{2}}{4} \int_{\Omega} d^{\alpha} \frac{\left|\nabla_{\mathbb{H}^{n}} \phi\right|^{2}}{d^{2}} d V+K(C, \alpha) \int_{\Omega} d^{\alpha-4} \frac{\phi^{2}}{\left(\ln \frac{R}{d}\right)^{2}} d V
$$

for all compactly supported smooth functions $\phi \in C_{0}^{\infty}(\Omega), \frac{8-n}{3}<\alpha<2$ and $K=$ $\frac{(n-\alpha)(n+3 \alpha-8)}{16}$

Corollary 3.2. Let $B_{R}$ be a hyperbolic ball with center 0 and hyperbolic radius $R$. Let $d=\log \left(\frac{1+|x|}{1-|x|}\right)$ and $\delta:=R-d$. Then the following inequality is valid:

$$
\int_{\Omega} d^{\alpha}\left|\Delta_{\mathbb{H}^{n}} \phi\right|^{2} d V \geq \frac{(n-\alpha)^{2}}{4} \int_{\Omega} d^{\alpha} \frac{\left|\nabla_{\mathbb{H}^{n}} \phi\right|^{2}}{d^{2}} d V+K(C, \alpha) \int_{\Omega} d^{\alpha-2} \frac{\phi^{2}}{(R-d)^{2}} d V
$$

for all compactly supported smooth functions $\phi \in C_{0}^{\infty}(\Omega), \frac{8-n}{3}<\alpha<2$ and $K=$ $\frac{(n-\alpha)(n+3 \alpha-8)}{16}$. 
Using the same argument as in the proof of Theorem 3.1 and improved HardySobolev type inequality (2.16), we obtain the following improved Rellich-Sobolev type inequality on the hyperbolic space $\mathbb{H}^{n}$.

Corollary 3.3. Let $\phi \in C_{0}^{\infty}\left(\mathbb{H}^{n}\right)$ and $d=\log \left(\frac{1+|x|}{1-|x|}\right)$. Then the following inequality is valid:

$$
\begin{aligned}
\int_{\mathbb{H}^{n}} d^{\alpha}\left|\Delta_{\mathbb{H}^{n}} \phi\right|^{2} d V \geq & \frac{(n-\alpha)^{2}}{4} \int_{\mathbb{H}^{n}} d^{\alpha} \frac{\mid \nabla_{\left.\mathbb{H}^{n} \phi\right|^{2}}}{d^{2}} d V \\
& +K\left(\int_{\mathbb{H}^{n}} d^{\frac{(n-2)(q-2)+(\alpha-2) q}{2}} \phi^{q} d x\right)^{2 / q},
\end{aligned}
$$

where $\frac{8-n}{3}<\alpha<2, K=\frac{(n-\alpha)(n+3 \alpha-8) 2^{n-2}}{4 c^{2}}\left(\frac{\left|\mathbb{S}^{n}\right|}{2}\right)^{\frac{q-2}{q}}, 2 \leq q \leq \frac{2 n}{n-1}+2 \epsilon, \epsilon>0$, and $c>0$.

\section{UNCERTAINTY PRINCIPLE INEQUALITY}

The first and most famous uncertainty principle goes back to Heisenberg's seminal work, which was developed in the context of quantum mechanics [15. It says that the position and momentum of a particle cannot be determined exactly at the same time but only with an "uncertainty". The mathematical version of this principle (stating that a function and its Fourier transform cannot be well localized simultaneously) was formulated afterwards by Pauli and Weyl [24] and it is sometimes referred to as the Heisenberg-Pauli-Weyl inequality. Uncertainty principle type inequalities are central to harmonic analysis and such considerations of the time-frequency domain are crucial in signal and image processing [10].

In a previous work [17, we obtained a Heisenberg-Pauli-Weyl inequality on a compete noncompact Riemannian manifold $M$ and found an explicit constant when $M$ is the hyperbolic space $\mathbb{H}^{n}$. In the present paper we first prove a HeisenbergPauli-Weyl inequality for general Riemannian manifolds which has a better constant than those of [17] and then we obtain the sharp constant in the hyperbolic case. The following is the first result of this section.

Theorem 4.1. Let $M$ be a complete Riemannian manifold of dimension $n \geq 2$. Let $\rho$ be a nonnegative function on $M$ such that $|\nabla \rho|=1$ and $\Delta \rho \geq \frac{C}{\rho}$ in the sense of distribution where $C>0$. Then the following inequality holds:

$$
\left(\int_{M} \rho^{2} \phi^{2} d V\right)\left(\int_{M}|\nabla \phi|^{2} d V\right) \geq \frac{(C+1)^{2}}{4}\left(\int_{M} \phi^{2} d V\right)^{2}
$$

for all compactly supported smooth functions $\phi \in C_{0}^{\infty}(M)$.

Proof. Using the assumptions $|\nabla \rho|=1$ and $\Delta \rho \geq \frac{C}{\rho}$, we get

$$
\int_{M}\left(\Delta \rho^{2}\right) \phi^{2} d V \geq(2 C+2) \int_{M} \phi^{2} d V .
$$

By integration by parts and the Cauchy-Schwarz inequality, we have

$$
\left(\int_{M} \rho^{2} \phi^{2} d V\right)\left(\int_{M}|\nabla \phi|^{2} d V\right) \geq \frac{(C+1)^{2}}{4}\left(\int_{M} \phi^{2} d V\right)^{2} .
$$

This completes the proof. 
We now prove a sharp analogue of the Heisenberg-Pauli-Weyl inequality (1.3) on the hyperbolic space $\mathbb{H}^{n}$.

Theorem 4.2. Let $\phi \in C_{0}^{\infty}\left(\mathbb{H}^{n}\right), d=\log \left(\frac{1+|x|}{1-|x|}\right)$ and $n>2$. Then

$$
\left(\int_{\mathbb{H}^{n}} d^{2} \phi^{2} d V\right)\left(\int_{\mathbb{H}^{n}}\left|\nabla_{\mathbb{H}^{n}} \phi\right|^{2} d V\right) \geq \frac{n^{2}}{4}\left(\int_{\mathbb{H}^{n}} \phi^{2} d V\right)^{2}
$$

Moreover, equality holds in (4.3) if $\phi(x)=A e^{-\alpha d^{2}}$, where $A \in \mathbb{R}, \alpha=\left(\frac{n-1}{n-2}\right)(n-$ $\left.1+2 \pi \frac{C_{n-2}}{C_{n}}\right)$ and $C_{n}=\int_{\mathbb{H}^{n}} e^{-\alpha d^{2}} d V$.

Proof. The inequality follows from Theorem 4.1. In order to achieve equality inspired by the Euclidean case, we consider hyperbolic analogues of Gaussians: $\phi(x)=A e^{-\alpha d^{2}}$ where $A \in \mathbb{R}$ and $\alpha>0$. A straightforward but tedious calculation shows that $\phi(x)=A e^{-\alpha d^{2}}$ is the minimizer where $\alpha=\left(\frac{n-1}{n-2}\right)\left(n-1+2 \pi \frac{C_{n-2}}{C_{n}}\right)$ and $C_{n}=\int_{\mathbb{H}^{n}} e^{-\alpha d^{2}} d V$.

Remark. Note that even though $\phi(x)=A e^{-\alpha d^{2}}$ does not have a compact support, it can be approximated by such functions yielding that (4.3) is sharp.

There is a natural link between Hardy, Heisenberg-Pauli-Weyl and Rellich type inequalities. For instance, using the Rellich-type inequality II (3.1), we have the following second-order Heisenberg-Pauli-Weyl inequality.

Theorem 4.3. Let $M$ be a complete Riemannian manifold of dimension $n \geq 2$. Let $\rho$ be a nonnegative function on $M$ such that $|\nabla \rho|=1$ and $\Delta \rho \geq \frac{C}{\rho}$ in the sense of distribution where $C>7$. Then the following inequality holds:

$$
\left(\int_{M} \rho^{4} \phi^{2} d V\right)\left(\int_{M}|\Delta \phi|^{2} d V\right) \geq \frac{(C+1)^{4}}{16}\left(\int_{M} \phi^{2} d V\right)^{2}
$$

for all compactly supported smooth functions $\phi \in C_{0}^{\infty}\left(M-\rho^{-1}\{0\}\right)$.

Proof. By equation (4.2) and the Cauchy-Schwarz inequality, we get

$$
\left(\int_{M} \rho^{4} \phi^{2} d V\right)^{1 / 2}\left(\int_{M} \frac{|\nabla \phi|^{2}}{\rho^{2}} d V\right)^{1 / 2} \geq \frac{C+1}{4} \int_{M} \phi^{2} d V .
$$

Using the Rellich-type inequality II (3.1), we obtain the desired inequality:

$$
\left(\int_{M} \rho^{4} \phi^{2} d V\right)\left(\int_{M}|\Delta \phi|^{2} d V\right) \geq \frac{(C+1)^{4}}{16}\left(\int_{M} \phi^{2} d V\right)^{2}
$$

As an immediate consequence of the Theorem 4.3 we have the following secondorder Heisenberg-Pauli-Weyl inequality with an explicit constant on the hyperbolic space $\mathbb{H}^{n}$. 
Corollary 4.1. Let $\phi \in C_{0}^{\infty}\left(\mathbb{H}^{n}-\{0\}\right), d=\log \left(\frac{1+|x|}{1-|x|}\right)$ and $n>8$. Then the following inequality holds:

$$
\left(\int_{\mathbb{H}^{n}} d^{4} \phi^{2} d V\right)\left(\int_{\mathbb{H}^{n}}\left|\Delta_{\mathbb{H}^{n}} \phi\right|^{2} d V\right) \geq \frac{n^{4}}{16}\left(\int_{\mathbb{H}^{n}} \phi^{2} d V\right)^{2} .
$$

\section{References}

[1] Adimurthi, M. Ramaswamy and N. Chaudhuri, An improved Hardy-Sobolev inequality and its applications, Proc. Amer. Math. Soc. 130 (2002), 489-505. MR1862130 (2002j:35232)

[2] W. Allegretto, Finiteness of lower spectra of a class of higher order elliptic operators, Pacific J. Math. 83, (1979), no.2, 303-309. MR557930 (81d:35063)

[3] G. Barbatis, S. Filippas and A. Tertikas A unified approach to improved $L^{p}$ Hardy inequalities with best constants, Trans. Amer. Math. Soc. 356 (2004), no. 6, 2169-2196. MR2048514 (2005a:26016)

[4] G. Barbatis, Best constants for higher-order Rellich inequalities in $L^{p}(\Omega)$, Math. Z. 255 (2007), no. 4, 877-896. MR.2274540 (2008c:46047)

[5] R.D. Benguria, R.L. Frank and M. Loss, The sharp constant in the Hardy-Sobolev-Maz'ya inequality in the three dimensional upper half-space, Math. Res. Lett. 15 (2008), no. 4, 613622. MR2424899 (2009j:46074)

[6] H. Brezis and J. L. Vázquez, Blow-up solutions of some nonlinear elliptic problems, Rev. Mat. Univ. Complutense Madrid 10 (1997), 443-469. MR1605678 (99a:35081)

[7] G. Carron, Inégalitès de Hardy sur les variétés riemanniennes non-compactes, J. Math. Pures Appl. (9) 76 (1997), no. 10, 883-891. MR1489943 (99c:53026)

[8] C. Cowan, Optimal Hardy inequalities for general elliptic operators with improvements. Commun. Pure Appl. Anal. 9 (2010), no. 1, 109-140. MR2556749 (2010m:35008)

[9] E. B. Davies, and A. M. Hinz, Explicit constants for Rellich inequalities in $L_{p}(\Omega)$, Math. Z. 227 (1998), no. 3, 511-523. MR 1612685 (99e:58169)

[10] G. B. Folland and A. Sitaram, The Uncertainty Principle: A Mathematical Survey, J. Fourier Anal. Appl. 3 (1997), 207-238. MR1448337 (98f:42006)

[11] E. Fabes, C. Kenig and R. Serapioni, The local regularity of solutions of degenerate elliptic equations, Comm. in P.D.E., 7 (1982), 77-116. MR643158 (84i:35070)

[12] N. Ghoussoub, A. Moradifam. On the best possible remaining term in the Hardy inequality, Proc. Natl. Acad. Sci. USA 105 (2008) no 37, 13746-13751. MR2443723 (2009i:26031)

[13] N. Ghoussoub, A. Moradifam. Bessel pairs and optimal Hardy and Hardy-Rellich inequalities, Math. Ann. 349 (2011), 1-57. MR2753796 (2011m:35012)

[14] G. Grillo, Hardy and Rellich-type inequalities for metrics defined by vector fields, Potential Analysis 18 (2003), 187-217. MR.1953228 (2003j:46043)

[15] W. Heisenberg, Über den anschaulichen Inhalt der quantentheoretischen Kinematik und Mechanik, Z. Physik 43 (1927), 172-198.

[16] E. Hebey, Nonlinear analysis on manifolds: Sobolev spaces and inequalities, CIMS Lecture Notes, Courant Institute of Mathematical Sciences, Vol. 5, 1999. Second edition published by the American Mathematical Society, 2000 MR 1688256 (2000e:58011)

[17] I. Kombe and M. Özaydin, Improved Hardy and Rellich inequalities on Riemannian manifolds, Trans. Amer. Math. Soc. 361 (2009), 6191-6203. MR2538592 (2010i:53061)

[18] P. Li and J. Wang, Weighted Poincaré inequality and rigidity of complete manifolds, Ann. Sci. École Norm. Sup. (4) 39 (2006), no. 6, 921-982. MR2316978 (2008d:53053)

[19] V. Minerbe, Weighted Sobolev inequalities and Ricci flat Manifolds, Geom. Funct. Anal. 18 (2009), no. 5, 1696-1749. MR2481740 (2010g:53064)

[20] F. Rellich, "Perturbation theory of eigenvalue problems", Gordon and Breach, New York, 1969. MR0240668(39:2014)

[21] E. Stein, "Harmonic Analysis, Real-Variable Methods, Orthgonality, and Oscillatory Integrals", Princeton University Press, Princeton, NJ. MR1232192 (95c:42002)

[22] A. Tertikas and N. Zographopoulos, Best constants in the Hardy-Rellich Inequalities and Related Improvements, Advances in Mathematics 209, (2007), 407-459. MR2296305 (2007m:26014) 
[23] C. Xia, The Caffarelli-Kohn-Nirenberg inequalities on complete manifolds, Math. Res. Lett. 14 (2007), 875-885. MR2350131 (2009a:53064)

[24] H. Weyl, The Theory of Groups and Quantum Mechanics. Dover Publications, New York, 1931.

Department of Mathematics, Faculty of Science and Letters, Istanbul Commerce University, Selman-1 Pak Cad. No: 2, Üsküdar, Istanbul, Turkey

E-mail address: ikombe@ticaret.edu.tr

Department of Mathematics, University of Oklahoma, Norman, Oklahoma 73019-0315

E-mail address: mozaydin@math.ou.edu 